\title{
LE DÉFI DU NOUVEAU ROMAN *
}

\author{
Márcia Arbex**
}

Résumé: Dans ce travail, il s'agira d'exposer quelquesuns des aspects essentiels de l'cuuve d'Alain RobbeGrillet, comme un exemple d'approche possible du récit contemporain. Ayant récemment publié trois ouvrages, l'écrivain a traversé le XXe siècle en laissant une influence durable sur toute une génération marquée par le nouveau roman. C'est ainsi qu'une étape importante de l'bistoire de la littérature française peut être retracée à partir de l'étude de ses textes, dont le renouvellement de formes est une constante. En outre, l'auteur s'intéresse à différentes catégories d'images, ce qui permet d'envisager le rapport entre l'écriture et d'autres systèmes sémiotiques comme le cinéma et la peinture.

Dans la préface intitulée Le point sur Robbe-Grillet? (1962) ${ }^{1}$, Roland Barthes dit que "considérée dans son développement et dans son avenir (qu'on ne saurait lui assigner), l'œuvre de Robbe-Grillet devient alors l'épreuve du sens vécu par une certaine société, et l'histoire de cette œuvre sera à sa manière l'histoire de

\footnotetext{
- Texto entregue para publicação em novembro de 2004.

" Professora da Faculdade de Letras/UFMG.

' Préface à Bruce MORRISSETTE (1963), publiée ultérieurement dans Essais critiques (1964).
} 
cette société" (1964:212). Aujourd'hui, nous nous confrontons encore à cette épreuve même si l'influence durable que l'œuvre a laissée sur toute une génération d'écrivains sensibles aux innovations proposées initialement par le nouveau roman est devenue incontestable. Étudier cette œuvre signifie retracer une étape importante de l'histoire récente de la littérature française, une fois que l'écrivain, ayant publié son premier ouvrage, Les Gommes, en $1953^{2}$, nous surprend en 2001 avec deux nouvelles publications : un roman intitulé La Reprise et un recueil de textes, Le Voyageur ; et encore, en 2002, le ciné-roman C'est Gradiva qui vous appelle.

Le choix de l'écrivain comme exemple à notre propos relève d'une sorte de défi. Considérés souvent comme "difficiles" ou peu accessibles, ses romans sont peu étudiés en cours de littérature, alors que l'œuvre, se déployant sur près d'un demisiècle, est sans doute l'une des plus importantes de la littérature contemporaine. Cela tient sans doute à l'innovation apportée par son écriture et les ruptures provoquées par rapport au récit dit traditionnel : mise en question du personnage de fiction et de la notion d'intrigue, affirmation de l'importance des procédés intertextuels et de réécriture - autant d'aspects essentiels qui ont au départ dérouté les premiers lecteurs, devraient être considérés non seulement dans l'étude de son œuvre en particulier, mais aussi comme des aspects importants devenus incontournables dans l'analyse du texte littéraire contemporain en général.

On pourra aborder l'œuvre par un autre biais, mais non des moindres: celui du rapport entre la littérature et d'autres

\footnotetext{
${ }^{2}$ Les Gommes (Paris: Éditions de Minuit, 1953), de fait, constitue sa première publication, étant donné qu' Un régicide est son premier livre, écrit en 1949 et publié seulement en 1978 aux Éditions de Minuit également.
} 
systèmes sémiotiques, en particulier le cinéma et la peinture, et redécouvrir ainsi une autre facette de l'écrivain. La peinture semble constituer un thème de prédilection auquel l'auteur a parfois recours pour faire voir au lecteur, au moyen de descriptions picturales, de citations de noms d'artistes ou d'œuvres d'art, l'image mise en mots des phantasmes les plus récurrents chez ses personnages, ou bien des références iconiques qui semblent peupler l'imagination, les rêves et la mémoire de l'écrivain. Le cinéma, enfin, domaine auquel Robbe-Grillet s'est consacré dès 1961, écrivant les scénarios et réalisant une dizaine de films considérés comme des films "d'auteur", lesquels ont d'abord dérouté, parfois choqué, mais dans tous les cas fasciné les spectateurs.

C'est à partir de l'exposé de quelques-uns des aspects essentiels de son ouvre que nous nous proposons de fournir un exemple d'approche possible du texte littéraire qui se donne comme point de départ le choix d'un écrivain autour duquel tout un "univers" littéraire et artistique se construit, tout un contexte historico-littéraire peut être reconstitué et étudié. Il ne s'agirait donc pas ici de procéder à une analyse minutieuse des idées théoriques et des romans ou scénarios de l'écrivain, ni de proposer des activités ou une méthodologie à suivre, mais de dégager des lignes d'approche qui puissent ouvrir l'accès à cet "univers".

\section{Définition du nouveau roman et procédés de rupture}

Le nom d'Alain Robbe-Grillet est intimement lié au nouveau roman, terme qu'il convient tout d'abord de définir : il ne s'agit pas, selon l'écrivain, d'un terme pour désigner une école, ni d'un groupe constitué d'écrivains qui travailleraient selon les mêmes principes ; il s'agirait plutôt d'une "appellation commode 
englobant tous ceux qui cherchent de nouvelles formes romanesques, capables d'exprimer (ou de créer) de nouvelles relations entre l'homme et le monde, tous ceux qui sont décidés à inventer le roman, c'est-à-dire, inventer l'homme" (PNR, p. 9) 3 . Cela veut dire que le nouveau roman ne cherche pas à développer des théories, même si ces écrivains affirment ne pas établir d'antinomie entre création et conscience, entre écriture et réflexion critique : "(..) on s'achemine de plus en plus vers une époque de la fiction où les problèmes de l'écriture seront envisagés lucidement par le romancier, et où les soucis critiques, loin de stériliser la création, pourront au contraire lui servir de moteur". (PNR, p.11). Dans le sens défini ci-dessus, le nouveau roman, à travers les œuvres d'Alain Robbe-Grillet, Michel Butor, Nathalie Sarraute, Claude Simon, Robert Pinget, entre autres, s'est caractérisé par de nombreuses innovations techniques et théoriques apportées au récit, et plus particulièrement au roman, innovations insérées dans le contexte historique et littéraire qui a été marqué par des réflexions profondes autour de la question du rôle de l'artiste, celui de la littérature et de ses moyens, comme nous le savons.

En France, la voie s'est ouverte aux expérimentations textuelles de ce groupe hétérogène d'auteurs, réunis parfois malgré eux sous le terme de "nouveaux romanciers", par la lecture de l'œuvre de certains écrivains comme Kafka ou Faulkner dont l'importance est aujourd'hui reconnue. Robbe-Grillet reconnaît ces influences déterminantes dans les sources du nouveau roman, en ajoutant celle de Roussel, de Joyce, des surréalistes et de Samuel Beckett.

${ }^{3}$ Alain ROBBE-GRILLET, Pour un nouveau roman, Paris: Éditions de Minuit, 1961. Les citations extraites de cet ouvrage essentiel seront désignées par les initiales PNR, suivies du numéro de la page. 
À la suite de ces devanciers, le questionnement sur l'écriture poétique évolue et se dirige premièrement contre le roman classique, c'est-à-dire, le roman français tel qu'il s'est développé au XIXe siècle : "La seule conception romanesque qui ait cours aujourd'hui est, en fait celle de Balzac", dénonce Robbe-Grillet en 1956, dans Une voie pour le roman futur (PNR, p.15-23). Suivant les pas d'André Breton dans le Manifeste du surréalisme (1924), il condamne la "sacro-sainte analyse psychologique", seule responsable de la conception du récit et des personnages, de même que du développement de l'intrigue; il attaque encore cet "univers des significations" que constitue le roman en proposant la "destitution des vieux mythes de la profondeur" et en affirmant la présence des objets :

“(...) non seulement nous ne considérons plus le monde comme notre bien, notre propriété privée, calquée sur nos besoins et domesticable, mais par surcroît nous ne croyons plus à cette profondeur. Tandis que les conceptions essentialistes de l'homme voyaient leur ruine, l'idée de 'condition' remplaçant désormais celle de 'nature', la surface des choses a cessé d'être pour nous le masque de leur cœur, sentiment qui préludait à tous les 'au-delà' de la métaphysique". (PNR, p.22)

Postérieurement, en 1958, dans le texte Nature, Humanisme, Tragédie (PNR, p.45), Robbe-Grillet reprend la discussion sur la nature humaine, en réponse à la polémique qui s'est créée et aux attaques reçus à propos de la négation de la "profondeur". C'est l'occasion pour l'auteur de réaffirmer sa conviction: refuser la prétendue "nature humaine", de même que le vocabulaire (métaphores, analogies) qui perpétue son mythe, et placer les objets comme purement extérieurs et superficiels ne signifie pas nier l'homme, mais "c'est repousser l'idée 
'pananthropique' contenue dans l'humanisme traditionnel, et probablement dans tout humanisme", en revendiquant sa propre liberté.(PNR, p.52).

La lecture de l'œuvre de Kafka et de celle de Faulkner, entre autres, a apporté la confirmation à ces "iconoclastes" - les nouveaux romanciers, de vouloir " réinterroger, par les moyens de la création et de la réflexion théorique, une réalité qui échappe aux critères habituels de l'intelligence rationaliste ou mystique" (ALLEMAND, 1997:14). Ces auteurs considèrent l'écriture comme la recherche d'un nouveau réalisme qui, au lieu de mimer le monde ou de é créer une illusion référentielle, se sont donnés pour but l'inventer, en posant "la particularité et la parcellisation de optique personnel comme seules garantes d'une quelconque authenticité de la représentation" (Ibidem : 15). Lisons Robbe-Grillet à ce propos:

"l'artiste contemporain sait qu'il n'a aucune parcelle de vérité à communiquer (...). C'est seulement quelqu'un qui a éprouvé le besoin d'inventer des formes. Et cellesci ne se présentent pas du tout comme étant la vérité, mais comme une architecture possible du monde, une organisation possible fragmentaire, provisoire et mouvante du monde par l'esprit créateur de l'homme." (apud ALLEMAND, 1997:15).

Cette nouvelle écriture cherche donc à briser les points d'ancrage au réel qui permettent la création d'une illusion référentielle réconfortante, d'une vision rassurante du réel. À partir du moment où l'artiste se refuse à produire un roman "crédible", à créer un univers saisissable, traduisible, cohérent et passible d'être dominé par l'écriture, où les personnages et les choses représentés auraient une "existence", à partir de ce moment le texte tend à ne plus se présenter comme miroir du 
monde et se donne comme travail d'écriture "ne renvoyant à rien d'autre qu'à lui-même". (Ibidem : 15)

Des procédés de rupture dont l'objectif serait la rénovation des formes du récit ont été identifiés dans toute l'œuvre de Robbe-Grillet. Certains sont plus facilement repérables dans celles considérées par la critique comme étant de la première période : d'Un Régicide à Dans le Labyrinthe, celle-ci établissant le passage avec les récits et les films postérieurs. Ils peuvent être résumés comme suit : la dilution du personnage, l'importance accordée aux objets, la dissolution de la notion d'intrigue et la dislocation de la diégèse. Dans la deuxième période", plus formaliste et ludique, "le matériau artistique devient le cœur problématique de la narration" et "l'amusement [est] ici indissociable des manipulations structurales d'œuvres qui exhibent la matérialité de leur art en jouant des stéréotypes littéraires (...)" (ALLEMAND, 1997:18). Les procédés "intra-intertextuels" sont également au cœur des préoccupations sur la construction du récit, comme le témoigne l'auteur dans l'entretien avec Jean-Jacques Brochier: "Comme si je n'avais écrit qu'un seul livre, qui aurait commencé avec Un Régicide, qui se poursuivrait encore aujourd'hui avec La Reprise, et qui raconterait une seule histoire, mouvante et aventureuse. Une histoire qui, parfois, a l'air de se répéter, mais qui se transforme sans cesse." (2001:24)

Dans l'étude de l'œuvre, ces procédés constituent les principaux points sur lesquels se heurtent les lecteurs en général, leur éclaircissement devenant ainsi une tâche majeure. Nous donnerons ensuite un aperçu de ces procédés en mettant en évidence surtout leur caractère innovateur.

\footnotetext{
${ }^{4}$ Une troisième période a été également proposée, celle composée par la trilogie des Romanesques (1984-1994) : Le Miroir qui revient, Angélique ou lenchantement, Les Derniers Jours de Corinthe.
} 


\section{La dilution du personnage}

Nous connaissons tous, du moins en partie, la déclaration provocante et célèbre de l'auteur : "Le roman de personnages appartient bel et bien au passé, il caractérise une époque : celle qui marqua l'apogée de l'individu. Peut-être n'est-ce pas un progrès, mais il est certain que l'époque actuelle est plutôt celle du numéro matricule."(PNR, p.28). Pour témoigner de son appartenance au monde contemporain, le roman se doit d'être moins anthropocentriste, renoncer à la toute-puissance de la personne et au culte exclusif de "l'humain", d'où la dilution de la figure du héros. Le personnage est alors rendu à son étymologie: "un masque (persona) et non un véritable individu, auquel il substitue un effet de personnage, c'est-à-dire un être dont la seule validité est dans sa nature linguistique, (...), tout juste assez caractérisé pour faire tenir la narration debout." (ALLEMAND, 1997 :16). Robbe-Grillet observait à propos des héros des récits de Samuel Beckett qu'ils se dégradaient de livre en livre, et de plus en plus vite, de sorte que "ces créatures, qui ont défilé sous nos yeux, elles ne servaient qu'à nous abuser ; elles occupaient les phrases du roman à la place de l'être insaisissable qui refuse toujours d'y paraitre, l'homme incapable de récupérer sa propre existence, celui qui ne parvient jamais à être présent."(PNR, p.95).

Si Le Voyeur possède bien un "héros" nommé, Mathias, ce n'est pas le cas de La Jalousie, dont seul un personnage du trio, l'homme marié (l'amant présumé) possède un prénom : Franck, tandis que la femme mariée est désignée par l'initiale A... et son mari n'est qu'un "narrateur absent qui observe tout et dont on ne peut déceler la présence en creux qu'à des faibles indices." (ALLEMAND,1997:74). Le soldat de Dans le labyrinthe, lui, est au départ une figure anonyme dont la capote militaire affiche 
un numéro matricule illisible: "cinq ou six chiffres noirs dans un losange rouge" (ROBBE-GRILLET, 1959:20) et, comme le mari de La Jalousie, le destinataire du paquet énigmatique qu'il porte pendant toute sa déambulation dans la ville enneigée, est un personnage complètement absent, malgré le fait qu'il soit l'un des principaux actants du récit. Ce nouveau héros surgit aussi chez Robbe-Grillet comme un "sujet multinominal et volontiers pseudonyme". Dans La Reprise (2001: 65), ce roman d'espionnage à Berlin détruite après la Deuxième Guerre mondiale, il est désigné soit par Henri Robin, H.R. dit aussi Ascher, Boris Wallon dit Wall, Franck Matthieu, Mathias, selon la pièce d'identité utilisée et l'évolution du récit. Rappelons aussi que certains de ces prénoms appartiennent d'ailleurs à des personnages des romans précédents.

Mais au-delà de la fluctuation de l'identité marquée par l'utilisation ou pas des prénoms, la mise en question du personnage de fiction passe par la récurrence des thèmes du dédoublement de l'identité, de la gémellité, aussi bien que par la modulation du pronom, comme l'observe Vidal à propos de Dans le labyrinthe: "Après les deux romans à la troisième personne [Les Gommes et Le Voyeur] et celui qui abolissait toute distance entre ' il' et 'je' [La Jalousie], formant une sorte de personne intermédiaire, Dans le labyrinthe pose le 'je' dès la première phrase puis le perd pour le 'il' qui, le roman terminé, s'efface à nouveau en 'je'". (1975:4) Dans ce dernier roman, d'ailleurs, à la fin du récit, les identités se confondent, les personnages se superposent de sorte que le lecteur ne peut pas affirmer si le "moi" qui le referme est le soldat, son camarade blessé au combat ou le médecin qui est venu à son secours, ce qui explique la remarque de Vidal : "Sans insister sur ces modulations du pronom, (...), on peut cependant relever ici l'absence totale de justification qui fait du 'je' la même personne qu'un personnage 
(le médecin) sous la plume de bien des critiques. C'est à l'avance, relire le roman ; remplir ce 'je' d'une présence que seule la fin du texte connaît." (lbidem).

Le thème du double permet également de démontrer l'instabilité du personnage de fiction, à travers les jeux de miroir, le recours au portrait et à l'autoportrait, comme l'on peut constater dans divers passages de La Reprise : au début du récit, lorsque le narrateur se trouve dans le train qui le conduit à Berlin : “(...) j'ai, pour la première fois depuis fort longtemps, aperçu cet homme que j'appelle mon double, pour simplifier, ou bien mon sosie, ou encore et d'une manière moins thẹâtrale : le voyageur" (2001: 9) ; ou bien un peu plus loin, quand la présence de ce personnage devient source d'inquiétude, mais dont la nature fictionnelle est vite rappelée: "Deux hypothèses sont envisageables : ou bien l'usurpateur est seulement quelqu'un qui me ressemble, tel un jumeau (...) ; ou bien le voyageur est vraiment moi, c'est-à-dire ma véritable duplication, et, dans ce cas... Mais non! Une pareille supposition n'est pas réaliste." (2001: 22). Le recours au miroir permet au narrateur de faire un portrait du personnage qui, malgré quelques précisions d'ordre physique, demeure celui d'un reflet invraisemblable, une image "fêlée", peu reconnaissable, défaite et "anonyme" :

“(...) Ascher (...) redresse son visage vers le miroir fêlé, au-dessus du lavabo. C'est à peine s'il se reconnaît : ses traits sont brouillés, ses cheveux hirsutes, et sa fausse moustache n'est plus en place; à demi soulevée du côté droit, elle pend, légèrement de travers. (...) Il se regarde ensuite à nouveau, et s'étonne devant cette figure anonyme, sans caractère, malgré une dissymétrie encore plus accentuée que d'habitude.(...)". (2001:48) 
On pourra parler également de dédoublement du narrateur, quand le récit est interrompu par des notes infrapaginales dont la voix narrative n'est pas la même que celle du texte qui la précède. Ce procédé métalangagier innovateur est évident dans La Reprise. A la note 8 , un deuxième narrateur se propose de "reprendre", de corriger le premier narrateur ${ }^{5}$ : Profitant de ce que notre agent perturbé est en train de se noyer dans le flot des imparfaits et des passés indéfinis, nous pouvons préciser ou rectifier certains points de détail dans le long dialogue qui précède"(2001:104), pour conclure à la note 9 que "l'auteur du problématique récit veut sans aucun doute accréditer chez son lecteur éventuel la thèse de l'empoisonnement (...)"(2001 : 105).

La thématique du dédoublement de l'identité se trouve ainsi imbriquée avec des procédés littéraires qui mettent en évidence la dissolution du personnage de fiction tel qu'il se présentait auparavant, c'est-à-dire, un personnage "construit" physiquement et psychologiquement de manière solide, visible et crédible.

\section{La dissolution de la notion d'intrigue}

De même que la dissolution du personnage, celle de l'intrigue peut se présenter comme un véritable obstacle à une première lecture. De même que le personnage, l'engagement, la forme et le contenu, l' "histoire" est l'une des notions considérées "périmées" par Robbe-Grillet. Toutefois, l'écrivain avertit que : "de même qu'il ne faut pas conclure à l'absence de l'homme sous prétexte que le personnage traditionnel est disparu, il ne faut pas assimiler la recherche de nouvelles

\footnotetext{
'C'est l'un des sens donné par l'auteur au titre de son ouvrage : la reprise c'est "le maître qui reprend l'élève", "reprendre, ici, c'est corriger" (Magazine littéraire, p.22)
} 
structures du récit à une tentative de suppression pure et simple de tout événement, de toute passion, de toute aventure." (PNR, p.32)

Cette dissolution de l'intrigue qui trouble plus d'un lecteur consiste, en vérité, à mettre en avant le "mouvement de l'écriture" plutôt que l'anecdote. Contraire à l'avis des amateurs et des critiques selon lesquels un roman serait "avant tout une histoire" et, par conséquent, le "vrai romancier" celui qui saurait "raconter une histoire" qui paraisse naturelle, spontanée, ressemblante, Robbe-Grillet affirme que l'écriture, comme toute forme d'art, est "une" intervention "et non simplement "un moyen, une manière" de raconter une histoire: "Ce qui fait la force du romancier, c'est justement qu'il invente, qu'il invente en toute liberté, sans modèle. Le récit contemporain a ceci de remarquable : il affirme délibérément ce caractère, à tel point même que l'invention, l'imagination, deviennent à la limite le sujet du livre." (Ibidem:30)

Cette inversion de valeurs vise à détruire l'image d'un "univers stable, cohérent, continu, univoque, entièrement déchiffrable" imposée par le récit traditionnel dont le but serait de "faire ressembler ce que l'on écrit aux schémas préfabriqués dont les gens ont l'habitude, c'est-à-dire à l'idée toute faite qu'ils ont de la réalité" (Ibidem). Ce que le nouveau romancier propose ce n'est donc pas la suppression de l'anecdote mais la destitution de son "caractère de certitude, sa tranquillité, son innocence". (Ibidem :32). ${ }^{6}$

"Ces affirmations se rapportent également au refus de la littérature "engagée", ou "didactique", à la suite de certains surréalistes et de Sartre, celui-ci ayant reproché Robbe-Grillet "de vivre dans un monde abstrait et détaché des réalités socio-politiques de son temps "(ALLEMAND, 1997 :93). Selon Robbe- 
Dans son premier roman, Un régicide (1949), il est aisé d'identifier une intrigue politico-policière mais impossible de parler objectivement de crime. De même dans Les Gommes (1953) l'intrigue policière est une véritable parodie du genre et se révèle "une enveloppe vide", car constituée autour d'un "crime inexistant", comme le dit Allemand (1997:50). La structure du Voyeur (1955) se déploie également autour d'un crime hypothétique, de même que celle de La Reprise, rédigé presque un demi-siècle après.

\section{L'importance accordée aux objets par la description}

Aussi, constate-t-on que la dissolution du héros et la désagrégation de l'intrigue s'accompagnent d'une prépondérance accordée aux objets. Prenons l'exemple du Voyeur. Roland Barthes remarquait à ce propos que "la fable recule, s'amenuise, s'anéantit sous le poids des objets. Les objets investissent la fable, se confondent avec elle pour mieux la dévorer" (1964:68). Si dans Les Gommes, Barthes observe que "le monde objectif était supporté par une intrigue d'ordre policier", dans Le Voyeur, "il n'y a plus aucune qualification de l'histoire", celle-ci tend au "point zéro" car l'acte qui la sous-tend (l'improbable meurtre commis par Mathias) est "blanchi", constitue un vide dans le

Grillet, "l'art ne peut pas être réduit à l'état de moyen au service d'une cause qui le dépasserait, celle-ci fût-elle la plus juste, la plus exaltante" (...) étant donné que "l'instant de la création ne peut que le ramener aux seuls problèmes de son art" ( $\mathrm{PNR}, 35)$. L'engagement serait alors, non pas de nature politique, mais "la pleine conscience des problèmes actuels de son propre langage, la conviction de leur extrême importance, la volonté de les résoudre de l'intérieur" : c'est sa seule chance de "demeurer un artiste et, sans doute aussi, (...) de servir un jour peut-être à quelque chose - peut-être même à la révolution". (PNR, p.39) 
récit et dans la mémoire de ce voyageur de commerce. Du crime le lecteur ne connaît presque rien, car les données de l'histoire sont réduites à quelques objets : une cordelette, un pieu, un pilier, des bonbons, des cigarettes, objets dont la répétition quasi obsessive ainsi que la "coordination progressive dessinent "sinon le crime lui-même, du moins la place et le moment du crime", faisant naître "la probabilité d'un usage meurtrier qui les rassemblerait tous" (Ibidem). D'où l'importance des descriptions des objets.

Les exemples d'objets privilégiés par la description sont nombreux, la gomme et le quartier de tomate dans Les Gommes étant des exemples types. Nous en avons choisi d'autres, moins cités par la critique : la cafetière de Trois visions réfléchies, l'une des nouvelles des Instantanés:

"La cafetière est en faïence brune. Elle est formée d'une boule, que surmonte un filtre cylindrique muni d'un couvercle à champignon. Le bec est un $S$ aux courbes atténuées, légèrement ventru à la base. L'anse a, si l'on veut, la forme d'une oreille, ou plutôt de l'ourlet extérieur d'une oreille; mais ce serait une oreille mal faite, trop arrondie et sans lobe, qui aurait ainsi le forme d'une 'anse de pot'. Le bec, l'anse et le champignon du couvercle sont de couleur crème. Tout le reste est d'un brun clair très uni, et brillant."(1962:10)

Dans le labyrintbe, la description de la bille de verre :

“(...) C'est une bille de verre ordinaire, d'environ deux centimètres de diamètre. Toute sa surface est parfaitement régulière et polie. L'intérieur est tout à fait incolore, d'une transparence absolue, à l'exception d'un noyau central, opaque, de la grosseur d'un petit pois. Ce noyau est noir et rond ; de quelque côté qu'on 
regarde la bille, il apparaît comme un disque noir de deux à trois millimètres de rayon (...)"(1959:142). ;

ou encore celle de l'anneau, dans Le Voyeur:

"C'était un huit couché : deux cercles égaux, d'un peu moins de dix centimètres de diamètre, tangeants par le côté. Au centre du huit, on voyait une excroissance rougeâtre qui semblait être le pivot, rongé par la rouille, d'un ancien piton de fer. Les deux ronds, de part et d'autre, pouvaient avoir été creusés à la longue, dans la pierre, par un anneau tenu vertical contre la muraille, au moyen du piton, et ballant librement de droite et de gauche dans les remous de la marée basse.(...) "(1955:17).

Contrairement à la description dans les romans dits "réalistes", dont la minutie et le caractère de syncrétisme sensoriel aboutissent à une profusion de significations, de symboles et de correspondances, chez Robbe-Grillet la description suppose un ordre unique de saisie du réel : la vue, l'objet devenant ainsi une "résistance optique" (BARTHES, 1964 :33). La description se révèle "anthologique" et "jamais allusive", tellement elle s'efforce d'épuiser l'objet au point de le détruire, dans son sens classique. Absorbé par "la nature optique" de l'objet, celui-ci décline toute fonction, toute substance, toute profondeur, et se constitue en un "itinéraire visuel", un "parcours optique" (ibidem: 34). L'épuisement de l'objet par la description se révèle en fin de compte "mystificateur", conclut Barthes. Car si l'auteur du Voyeur situe l'objet de façon exagérée, démontre son obsession par la topographie, démultiplie les indications et précisions comme à droite, à gauche, comme s'il s'agissait de donner des repères "réalistes" à un spectateur fictif, son but serait de détruire l'espace traditionnel, en perturber les fondations, en 
lui substituant un nouvel espace doté d'une "profondeur temporelle ". (Ibidem: 39)

\section{La dislocation de la diégèse}

L'isolement de la description d'un objet peut faire ressortir également comment s'opère la dislocation de la diégèse, comme nous pouvons l'observer dans les divers passages de La Jalousie où il est question du scutigère.

Le récit est déplacé en fragments narratifs à chaque fois plus détachés les uns des autres, créant une circularité des scènes qui se veut reflet de la circularité du temps et de l'espace, en opposition à l'acception de la linéarité chronologique du récit, à l'axe unique qui visait assurer la cohérence narrative et sa prétendue authenticité.

Ces fragments sont organisés selon une structure narrative à géométrie variable marquée par des déboîtements, des collisions, des interférences, des répétitions et des remboîtements. Cette structure repose sur des thèmes récurrents ou des images leitmotive qui s'alternent, qui s'intercalent, dans un va-et-vient répétitif mais progressif tout de même, car ces retours apportent au récit des modifications parfois à peine perceptibles. Ce que la fragmentation du récit et son agencement "incohérent" révèlent également c'est la continuité du temps intérieur, du flux de conscience, le travail de la mémoire qui néglige les notions de passé, présent et futur.

Par ce procédé, il s'opère une dissolution de la référentialité. L'espace est éclaté par de confusions d'ordre optique; de jeux de miroir et de multiples mises en abyme, surtout dans les ouvres de la deuxième période et dans les films, de sorte qu'il n'y ait pas de points de repères stables. "C'est le reflet qui constitue la réalité, elle n'existe qu'à travers lui et ne renvoie 
qu'à sa propre apparence". (ALLEMAND, 1997:21) Il n'est pas étonnant que les tableaux de peinture, les photographies, les affiches ou d'autres types d'images ainsi que les statues et les poupées deviennent des instruments de cette mise en abyme dont le but serait de brouiller la référentialité ; ou alors les images se présentent comme des véritables moteurs de l'écriture.

\section{Les enjeux littéraires: un auteur et un cinéaste à redécouvrir}

Les procédés décrits ci-dessus de façon succincte, en exemple d'un travail de renouvellement des formes narratives, littéraires et cinématographiques, constituent un apport littéraire majeur qui a marqué l'œuvre de nombreux écrivains de la deuxième moitié du XXe siècle. Faire redécouvrir l'œuvre de Robbe-Grillet constitue, certes, un défi, mais les enjeux littéraires et sémiotiques qu'elle propose en font le mérite.

De même que le romancier, le cinéaste Robbe-Grillet opère un détournement de l'illusion mimétique, procède à l'abandon à chaque fois plus radical des règles de la représentativité (VIDAL, 1975 :6). Le personnage féminin - soit dans les films, soit dans les romans - devient alors un élément privilégié de ce processus de rupture avec la mimèsis traditionnelle à laquelle se substitue "un effet d'étrangeté où les sujets animés sont réifiés, tels de petits objets mécaniques qui ne servent que de rouages intermédiaires entre le texte et son lecteur "(ALLEMAND, 1997:20). D'où l'émergence de cet aspect essentiel du cinéma de RobbeGrillet, déjà assez présent dans ses romans, qui est l'érotisme comme "une forme de fétichisme doublé d'une fascination pour l'artefact"(PEETERS, 2001:57).

Ce détournement de l'illusion mimétique aboutit en fin de compte, comme le suggère Allemand, à une interrogation "sur le statut même des différents niveaux de la diégèse qui (...) 
semblent faire coexister deux niveaux de fiction : la fiction première (en gros, celle de l'histoire) et la fiction seconde, qui relève non plus d'un récit linéaire mais d'une réalité mentale qui procède par bouffées d'imaginaire." (1997:108) Cette interrogation prendra forme aussi dans la trilogie des Romanesques (19841994), récits donnés comme autobiographiques mais dont la distance avec l'introspection, l'authenticité et la réflexion qui sont ces caractéristiques conventionnelles, en fait un nouveau "genre", comme le confirme Robbe-Grillet dans son entretien avec Jean-Jacques Brochier:

"De mon point de vue, il n'y a pas de différénce entre les Romanesques et mes romans. Je les aurais aussi bien sous-titrées 'roman' et si Le Miroir qui revient, le premier volume des Romanesques, est relativement acceptable du point de vue autobiographique, dès le second volume ça se gâte, et dans le troisième ça devient complètement de la fiction" (2001:21).

Des faits vécus y sont en effet évoqués, mais de façon discontinue et hétérogène ; la mémoire n'est pas fidèle, il y a des failles, les faits supposés vrais sont racontés comme s'il s'agissait de fables. Le genre est "subverti" et "la vérité est ce qui fait illusion" (ROUDAUT, 2001:52). L'enjeu consiste dans la rupture des frontières entre la fiction et la non-fiction, dans l'abolition des limites entre le vrai et le faux, dans l'effacement du cadre qui sépare un dehors d'un dedans - comme nous le propose aussi la série de trompe-l'œil de Magritte intitulés La Belle Captive ${ }^{7}$-, dans l'interrogation, enfin, de ce que c'est écrire - et lire.

${ }^{7}$ La Belle Captive, 1931, Hogarth Galleries, Sydney ; et la Belle Captive, 1948, Collection particulière ; Belle Captive, 1967, Communauté Française de Belgique. 
Resumo: Neste trabalbo, trata-se de expor alguns aspectos essenciais da obra de Alain Robbe-Grillet como exemplo de abordagem da narrativa contemporânea. Tendo puibicado recentemente três livros, o escritor francês atravessou o século XX deixando uma influência efetiva em toda uma geração marcada pelo nouveau roman. Desta forma, uma etapa importante da bistoria da literatura francesa pode ser retraçada a partir do estudo de seus textos, cuja renovação das formas literarias é uma constante. Além disso, o autor se interessa por diversas categorias de imagens, permitindo abordar a relação entre escrita $e$ outros sistemas semioticos como o cinema e a pintura.

\section{Références bibliographiques}

ALLEMAND, Roger-Michel. Alain Robbe-Grillet. Paris : Éditions du Seuil, 1997

BARTHES, Roland. Essais critiques. Paris : Éditions du Seuil, 1964.

BROCHIER, Jean-Jacques. La Reprise du Nouveau Roman. Magazine littéraire, $\mathrm{n}^{\circ} 402$, octobre 2001 , p.21-26.

LOUVEL, Liliane. Texte/image. Images à lire, textes à voir. Rennes : Presses Universitaires de Rennes, 2002.

MORRISSETTE, Bruce. Les romans de Robbe-Grillet. Paris : Les Éditions de Minuit, 1963.

PEETERS, Benoît. Un cinéaste à redécouvrir. Magazine littéraire, $\mathrm{n}^{\circ} 402$, oct. 2001, p.57-60.

ROBBE-GRILLET, A. Pour un nouveau roman. Paris : Les Éditions de Minuit, 1961.

ROBBE-GRILLET, A. Instantanés. Paris : Les Éditions de Minuit, 1962.

ROBBE-GRILLET, A. Dans le labyrinthe. Paris : Les Éditions de Minuit, 1959.

ROBBE-GRILLET, A. Cest Gradiva qui vous appelle. Paris : Les Éditions de Minuit, 2002 . 
ROBBE-GRILlET, A. La Reprise. Paris : Les Éditions de Minuit, 2002.

ROBBE-GRILLET, A. La belle captive. Lausanne, Paris : La bibliothèque des arts, 1976.

ROUDAUT, Jean. Le cercle autobiographique. Magazine littéraire, $n^{\circ} 402$, oct. 2001, p.52-55.

VIDAL, Jean-Pierre. Dans le labyrinthe, de Robbe-Grillet. Paris : Librairie Hachette, 1975.

VOUILLOUX, Bernard. La peinture dans le texte: XVIIre-XXe siècles. Paris: CNRS Éditions, 1994. 